\title{
The possible interrelation of trans - Neptunian objects and long - period comets with the high-precision orbits
}

\author{
Ayyub Guliyev ${ }^{1}$, Shaig Nabiyev ${ }^{1,2}$ \\ ${ }^{1}$ Department of Planetary Science, Shamakhy Astrophysical Observatory Named after N. Tusi NAS, Y. Mammadeliyev Distr. Shamakhy \\ r., Azerbaijan \\ ${ }^{2}$ Department of Planetary Science, Shamakhy Astrophysical Observatory Named after N. Tusi NAS and Department of Physics, Qafqaz \\ University, Qafqaz University Campus, Xirdalan c/Baku, Azerbaijan
}

\section{Email address:}

ayyub54@yahoo.com (A. Guliyev), snebiyev@qu.edu.az (Sh. Nabiyev)

\section{To cite this article:}

Ayyub Guliyev, Shaig Nabiyev. The Possible Interrelation of Trans - Neptunian Objects and Long - Period Comets with the HighPrecision Orbits. American Journal of Astronomy and Astrophysics. Vol. 2, No. 4, 2014, pp. 38-41. doi: 10.11648/j.ajaa.20140204.11

\begin{abstract}
The question on the relationship of long-period comets (LPCs) with trans-Neptunian objects (TNOs) having the diameter more than $200 \mathrm{~km}$ is investigated. We analyze the distribution values of minimum orbit intersection distances (MOID) for 91 TNOs and 495 long-period comets (LPCs) with known initial orbits. The parameter $t$ - distribution characterizing the level of redundancy MOID for each selected planetary body is prefaced. In the case when MOIDs values are less than $1 \mathrm{AU}, 14$ values of thave significance, greater than 0.95, and for the case MOIDs less than 0.5 AU, 6 values of $\mathrm{t}$ have significance, more than 0.95 , and the ratio of $\mathrm{t}$ makes 56:35 and 50:41, accordingly. A reliable correlation no matter it is weak, between values $t$ and $I$ (inclination) is found out. The obtained these results indicate the assumption of possible relation of long-period comets with TNOs.
\end{abstract}

Keywords: Comets, General, Kuiper Belt, General, Celestial Mechanics

\section{Introduction}

The present article is a logical continuation of our two previous papers $[1,2]$ in which statistical aspects of the hypothesis about the relation of some parts of comets with trans-Neptunian objects are investigated. We recall the methodology and the main results of the studies cited above. In the first paper [1] the distribution of LPCs orbits with distant nodes nearby 60 known TNOs orbits of the largest diameter has been investigated.

It has been established by testing method that near the orbits of some TNOs especially, Orcus, Salacia (120347), 2002 AW197 (55565), $2000 \mathrm{CN} 105$ and 2007 OR10 (225088) and the number of distant cometary nodes significantly exceed the background values. The short explanation will be presented below.

Analogously, the same method performed in the second paper [2] for the values of MOID in the comet-TNO system. The same data for 1048 LPCs and 91 TNOs having the largest diameters was used. The relation of two body categories is considered MOID in the comet-TNO system as an indicator [3]. The performed testing method showed that the number of MOID within less than $1 \mathrm{AU}$ and 0.5
AU for 13 and 9 TNO exceeds the background value respectively. The background values for all MOID were calculated during testing. The more interesting thing concerning the judgment of the calculations in this context is the following results: 2010 VZ98; 2001 UR163; 2003 AZ84; 2004 UX10; 2011 FW62; 2001 KA77; 2002 XV93; 2007 HV90; 2002 GJ32; 2005 CB79; 2007 XV50; 2008 AP129; 2002 XH91.

The methods and objects of the research remain almost the same in this paper as well as in [2], a difference is only in used material on cometary data.

The objects of the research are 91 TNOs with the diameters greater than $200 \mathrm{~km}$, and 495 long-period comets with known "original" values of the parameter $1 / a$. The cometary data is taken from the catalog [7], and the data for TNOs was taken from the official website of the IAU [5].

\section{Statistical Modeling}

The aim is to prove the degree of redundancy in the area of comets passing traffic TNOs in this case more than when the calculations are carried out regardless of their accuracy. It will serve it, as a point of comparison data in which it's 
meaning will be described below. As in the paper [2], after calculations, we will explore MOID zones corresponding to a distance of $1 \mathrm{AU}, 0.5 \mathrm{AU}$ and $0.1 \mathrm{AU}$ the orbit chosen TNO. Our expect for this research from the mechanism which provides the foundation of [1] and [2] is that most accurate cometary data should give higher values of the parameter $t$ (normalized difference) than in paper $[1,2]$.

In the first phase, we evaluate and analyze the values for 495 long-period comets with known "original" $1 / a$. Here $a$ refers to the "original" semi-major axis that the comet had before entering the planetary orbit. The frequencies are compared with the frequencies obtained in before [2]. In the second stage the "traffic zone" selected TNOs by the number of comet passes compared with 67-modeled zones [3], differing from it only in the parameters $\Omega$ (longitude of the ascending node) and I (inclination). We varied the parameters $I$ and $\Omega$ in such a way that the poles of the corresponding planes on the celestial sphere were distributed uniformly. In other words, to perform a comparative analysis, we substituted the specific planets with the virtual ones. Only after the comparison with them could we judge whether or not there was an excess of comets that closely approached to the orbit of a particular planet. In this case, $\Omega$ varies from $0^{\circ}$ to $330^{\circ}$ in steps by $30^{\circ}$, and I - from $0^{0}$ to $90^{\circ}$ with steps to the pole corresponding planes are equidistant from each other (Table 1). To compare difference $\mathrm{N}$ - number of comet passes in "traffic zone" having MOID less than fixed value regarding to the selected TNO with the average comet passes for 67 zones used Student's t- test formula.

$$
t=\frac{N-\bar{n}}{\sigma}
$$

Where $\bar{n}$ is the mean value of the data in the table, $\sigma$ is the root - main - square deviation (RMSD), $t$ is the normalized difference, and $\alpha$ is its confidence level determined from Student's distribution. As it is known, this distribution can be used only if the data of the table is random variables or as a whole is close to a normal distribution. Each time the interval $(\min N$; $\max N)$ was divided into seven parts (according to the Sturge's rule at the number of planes equal to 67) and the theoretical normal distributions were calculated by using a technique described by [4]. At a significance level of 0.05 , their deviations from the observed ones were within the randomness limits.

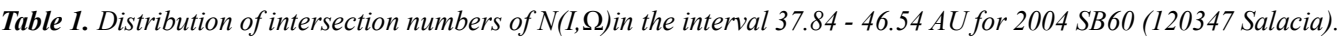

\begin{tabular}{|c|c|c|c|c|c|c|c|c|c|c|c|c|}
\hline $\mathrm{I} / \Omega(\mathrm{deg})$ & $\mathbf{0}$ & 30 & 60 & 90 & 120 & 150 & 180 & 210 & 240 & 270 & 300 & 330 \\
\hline 0 & 7 & & & & & & & & & & & \\
\hline 9,59 & 7 & 2 & 7 & 3 & 5 & 5 & 3 & 3 & 5 & 4 & 1 & 3 \\
\hline 19,47 & 2 & 3 & 7 & 4 & 1 & 0 & 5 & 5 & 1 & 2 & 2 & 2 \\
\hline 30 & 4 & 3 & 1 & 4 & 1 & 5 & 0 & 1 & 3 & 2 & 4 & 6 \\
\hline 41,81 & 2 & 3 & 3 & 5 & 3 & 5 & 2 & 3 & 5 & 4 & 0 & 4 \\
\hline 56,44 & 4 & 1 & 3 & 2 & 5 & 1 & 4 & 4 & 2 & 4 & 4 & 4 \\
\hline 90 & 0 & 4 & 3 & 3 & 4 & 1 & & & & & & \\
\hline
\end{tabular}

To apply the above-mentioned method to this table: $\mathrm{N}=7 ; \bar{n}=3.206 ; \sigma=1.758 ; \mathrm{t}=2.158 ; \alpha>0,98$ where $\mathrm{N}$ (I, $\Omega$ ) - number of comets intersection in $(0,0)$ plane, $\bar{n}$ - mean value of the table, $\sigma$ - root - main -square deviation, $\mathrm{t}$ - normalized difference, $\alpha$ - confidence level

\section{Minimum Orbit Intersection Distance (MOID) For TNOs}

To calculate the value of $\mathrm{r}$ (i.e. MOID) used the following formula:

$$
r=\left(R^{2}(d n)+\left[\frac{q(1+e)}{1+e \cos v}\right]^{2}-2 R(d n) \frac{q(1+e)}{1+e \cos v} \sqrt{1-\sin ^{2} I \times \sin ^{2}(\omega-v)}\right)^{\frac{1}{2}}
$$

Here $\mathrm{R}(d n)$ is the distance TNO in the direction of the ascending node corresponding comet's orbit, $q$ and $e$-nonvariant elements comet's orbits, $I$ and $\omega$ angular elements comet's orbits, $v$ - true anomaly of the comet, which $v$ varies from $0^{\circ}$ up to $360^{\circ}$ with the interval of $1^{0}$, and a minimum value selected from 360 values of $r$. Note that the last three elements are respect to the orbital plane of TNO but not to the ecliptic, and the starting point is its ascending node. The formula is derived from basic transformations of spherical triangles and requirements of finding a scalar vector magnitude TNO - comet in the triangle Sun - TNO comet [6]. The approximations of the given procedure consist of a small piece in a direction of the distant node of a planet considered circular. The formula of MOID can be determined from the other and more precisely methods however, considering a statistical property of research is possible to suppose, but this approximation does not influence to the results. We calculate MOIDs values for the asteroids relatively to the Earth, to test the abovementioned MOID's calculation formula, comparing them with values listed in [8], just only $3-5 \%$ of the errors interval found out.

Thus, the first stage of our calculations for 91 TNOs and 495 LPCs values $r$ (only 45045 values) are calculated. The 
initial comparison with the data of [2] can be carried out on the information contained in Table 2 . The values of the parameter $\mathrm{N}_{1}$ were taken from the paper of [2] for 1048 LPCs, and $\mathrm{N}_{2}$ is the number of MOIDs for 495 comets with high-precision orbits, in intervals for $0.05,0.1,0.2,0.5$ and 1 AU. In all considered cases, we can see the increase of $\mathrm{N}_{2}$ to $\mathrm{N}_{1}$ in percentage from $9 \%$ to $42 \%$ relatively. It means that we have a fair solid confirmation for the ideas contained in [2], that the results would increase when the increasing accuracy of the observed comets orbit parameters will find out.

Anyway, to determine the degree of redundancy of cometary intersections in zones of movement TNOs we use an additional the second method of comparison that requires large computational resources.

Table 2. The comparison of the comets numbers $N_{I}$ (for 1048 LPCs) and N2 (for 495 LPCs) in the different intervals of $r$ (MOID).

\begin{tabular}{llll}
\hline Interval $\mathbf{r}, \mathbf{A U}$ & $\mathbf{N}_{\mathbf{1}}(\mathbf{\%})$ & $\mathbf{N}_{\mathbf{2}}(\mathbf{\%})$ & Increment \\
\hline$<0.05$ & $11(0.011)$ & $6(0.013)$ & 1.18 \\
$<0.1$ & $32(0.033)$ & $21(0.047)$ & 1.42 \\
$<0.2$ & $105(0.109)$ & $55(0.122)$ & 1.19 \\
$<0.5$ & $552(0.572)$ & $282(0.626)$ & 1.09 \\
$<1$ & $2077(2.154)$ & $1068(2.371)$ & 1.10 \\
\hline
\end{tabular}

\section{The Results of Calculations for the Case $r<1$ AU}

The calculations for MOIDs of 91 TNOs and 495 LPCs show that 14 values of $t$ are likely significant, greater than 0.95 . There is a slight increase compared with the results in [2]. In this case, a significant gap between positive and negative values of $t$ is stored as a ratio of 56:35. Moreover, the obtained coefficient of correlation between the values of $t$ and $I$ is equal

$$
K(t, I)=-0.36 \pm 0.09 .
$$

The correlation coefficient value may seem is weak however, there is a methodology in a probability theory to assess its significance based on the criteria of the Student's [2]. It is common to test the correlation coefficient for how statistically significant might be and needs to calculate the root-mean-square error of the correlation coefficient:

$$
\operatorname{RMSE}=\left(1-0.36^{2}\right) /(91-1)^{0.5}=0.091
$$

Therefore, we have to compare the value of the ratio $\mathrm{h}=\mathrm{K} / \mathrm{RMSE}=3.92$ with the critical value. At a confidence interval of 0.01 , it is more than the critical value of 2.37 . It means that the correlation coefficient has a high significance. Take into account that it is not strongly dependent only on $\mathrm{K}$ but also on the number of TNO. If we had calculations 15 TNOs relatively, this significance would be within the randomness limits.

$\mathrm{K}$ is slightly less than in [2]. This relation is graphically reflected in a Fig. 1. Consequently, the application of finer material results of [2] remains valid. However, our attempt to get some relations between the values of $\mathrm{t}$ and $\mathrm{H}$ (absolute magnitude of TNO) was failed.

Probably, throughout interaction with comets, the weights and sizes do not play a dominant role and affinity of the movement of TNOs to ecliptics. Anyhow, this question needs the further check.

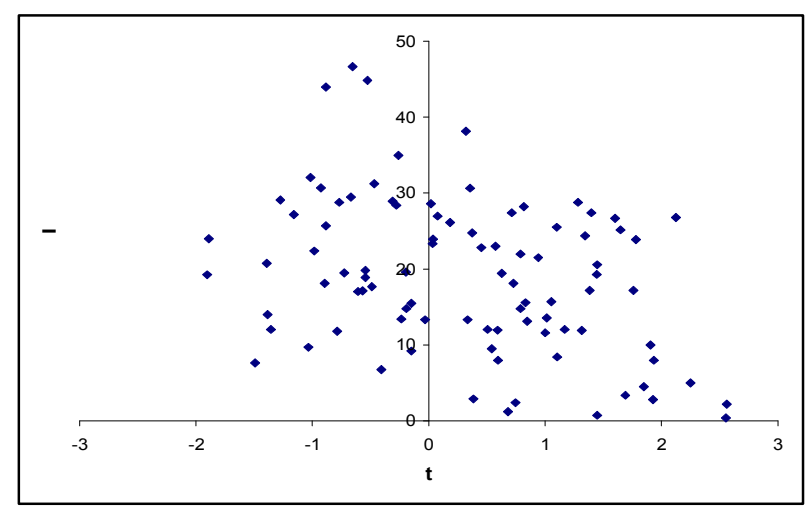

Fig 1. The relation between the values of $t$ and I for 495 long-period comets and 91 TNO in the case $r<1 \mathrm{AU}$.

\section{The Calculation Results for the Cases $r<0.5$ AU and $r<0.1$ AU}

The calculations show that in the case $\mathrm{r}<0.5$ AU only 6 values of $\mathrm{t}$ are likely significant, more than 0.95 . The above-described ratio in this case looks like 50:41, i.e. in this case, the regularities found in [2], remain valid. The correlation between the values of $\mathrm{t}$ and $\mathrm{I}$ is weak, but reliable

$$
\mathrm{K}(t, I)=-0.35 \pm 0.09
$$

For this case also $\mathrm{h}=3.89$ is higher than critical 2.37. And the good results obtained for the case $r<0.1 \mathrm{AU}$, in 8 cases it appears more than 1.85 including in 5 cases it is more than 2.2. In all other cases this parameter is always negative, but practically does not differ from zero (in the interval 0.5 to 0 ). We once again emphasize that this restriction for parameter $r$ is more rigid and apparently does not correspond to a reality if to consider that accuracy of the data on TNOs are not too high.

\section{Conclusion}

1. If to put high requirements concerning accuracy cometary data an obvious disproportion in distribution $t$ in favor of number of positive values as a whole remains.

2. If to compare the results in this paper for 495 comets which are well-known "original" semimajor axis or high-precision orbit parameters, with obtained for 1048 total LPCs in [2], in the cases $r<$ $1 \mathrm{AU}$, and $\mathrm{r}<0.5 \mathrm{AU}$ correlations were generally preserved.

3. All obtained results in this paper as previously 
getting results in $[1,2]$, once again indicate the hypothesis of the dynamic or other kind of relationship between LPCs and TNOs.

4. Furthermore, it is necessary to repeat the calculations with the application of new real restrictions of the cometary data. At the following stage of the given series investigations, authors plan to consider so-called hyperbolic comets particularly, with above-describe analogical method.

\section{References}

[1] Guliyev A.S, Guliyev R.A, 2011. On the question of relation of trans-Neptunian objects and comets. Azerbaijan Astronomical Journal. Baku, v. 6, n.4, p. 5

[2] Guliyev A.S, Nabiyev Sh. A, Guliyev R.A, Dadashov A.S,
2012. On the question of relation of comets to Kuiper bodies. Azerbaijan Astronomical Journal. Baku, v. 7, n.2, p. 5

[3] Guliyev A.S., 2007. On the Relationship of Long-Period Comets to Known and Unknown Planets. Astronomy Letters, v.33, n.8, p.562

[4] Gmurman V. E., 1968. Fundamentals of Probability Theory and Mathematical Statistics, American Elsevier Publishing Co., New York. p. 249

[5] http://minorplanetcenter.net/iau/lists/TNOs.html

[6] Kuz'michev V. V.,Tomanov V. P., 2006. Pis'ma Astron. Zh. Moskow,v.32, p.392

[7] Marsden B.G, Williams G.V, 2008. Catalogue of Cometary Orbits, $17^{\text {th }}$ edition, IAU, Central Bureau for Astronomical Telegrams, Cambridge, p.197

[8] http://www.minorplanetcenter.org/iau/lists/Dangerous.html 